\title{
A METRICAL PROPERTY OF POINT-SET TRANSFORMATIONS*
}

\author{
BY DEANE MONTGOMERY $†$
}

It is the purpose of this note to prove a theorem concerning distances between points of a metric spaceł under transformations of the space into itself. The distance between two points $p$ and $q$ of a metric space is denoted by $d(p, q)$. The point into which a point $p$ is taken by $n$ applications of a transformation $T$ is denoted by $p_{n}$, that is, $T$ takes $p$ to $p_{1}, p_{1}$ to $p_{2}$, and so on. If $d(p, q)$ is less than, greater than, or equal to $d\left(p_{1}, q_{1}\right), T$ is said, respectively, to increase, decrease, or to leave invariant the distance between $p$ and $q$. The theorem of this note shows that any continuous transformation of a suitably restricted metric space into itself leaves invariant the distance between some two distinct points of the space. In particular, it follows from the theorem that any continuous transformation of a closed or open $n$-sphere into itself leaves the distance between two distinct points of the sphere invariant. The theorem also applies to spherical surfaces.

It should be observed that in the following lemma the transformation is not assumed to be continuous as it is in the theorem. In both cases the phrase "into itself" means that the space goes in to the whole of itself and not into a proper subset of itself.

Lemma. If $E$ is a conditionally compact metric space and if $T$ is any one-to-one transformation of $E$ into itself which increases the distance between two points of $E$, then $T$ must also decrease the distance between two points of $E$.

Let $p$ and $q$ be two points of $E$ whose distance is increased by $T$, that is, $d(p, q)<d\left(p_{1}, q_{1}\right)$. The proof will be made by contradiction. Suppose that the lemma is false and that, for every two points $x$ and $y$ of $E$, we have $d(x, y) \leqq d\left(x_{1}, y_{1}\right)$.

* Presented to the Society, March 30, 1934.

$\dagger$ National Research Fellow.

$\ddagger$ For definitions of metric spaces and terms concerning them see Hausdorff, Mengenlehre, 1927. 
Assume first that neither $p$ nor $q$ is a periodic point of the transformation. Since $E$ is conditionally compact, the sequence $\left(p, p_{1}, p_{2}, \cdots\right)$ must have a subsequence $\left(p_{k_{1}}, p_{k_{2}}, p_{k_{z}}, \cdots\right)$ which satisfies the Cauchy convergence criterion. The sequence $\left(q_{k_{1}}, q_{k_{2}}, q_{k_{3}}, \cdots\right)$ must have a subsequence $\left(q_{n_{1}}, q_{n_{2}}, q_{n_{8}}, \cdots\right)$ which satisfies the Cauchy convergence criterion. Since $\left(n_{1}, n_{2}, n_{3}, \cdots\right)$ is a subsequence of $\left(p_{1}, p_{2}, p_{3}, \cdots\right)$, the sequence $\left(p_{n_{1}}, p_{n_{2}}, p_{n_{3}}, \cdots\right)$ also satisfies the convergence criterion. Therefore, for any positive number $\epsilon$ there is an $N$ such that for $n_{i}>N$ and $n_{j}>N$,

$$
d\left(p_{n_{i}}, p_{n_{j}}\right)<\epsilon \text {, and } d\left(q_{n_{i}}, q_{n_{j}}\right)<\epsilon .
$$

Choose $n_{i}$ and $n_{j}$ so that $n_{j}>n_{i}$. Then according to the supposition that the lemma is false and to the above inequalities,

$$
d\left(p, p_{n_{j}-n_{i}}\right) \leqq d\left(p_{n_{i}}, p_{n_{j}}\right)<\epsilon,
$$

and

$$
d\left(q, q_{n_{j}-n_{i}}\right) \leqq d\left(q_{n_{i}}, q_{n_{j}}\right)<\epsilon .
$$

Let $n_{j}-n_{i}$ be denoted by $r_{1}$. It can be seen that there is a sequence $\left(r_{1}, r_{2}, r_{3}, \cdots\right)$ such that $\left(p_{r_{1}}, p_{r_{2}}, p_{r_{3}}, \cdots\right)$ converges to $p$ and $\left(q_{r_{1}}, q_{r_{2}}, q_{r_{3}}, \cdots\right)$ converges to $q$. Hence for any $\epsilon$, the integer $i$ may be so chosen that $\left|d\left(p_{r_{i}}, q_{r_{i}}\right)-d(p, q)\right|<\epsilon$. In particular, there is some $i$ such that $\left|d\left(p_{r_{i}}, q_{r_{i}}\right)-d(p, q)\right|$ $<d\left(p_{1}, q_{1}\right)-d(p, q)$. This contradicts the fact that $d\left(p_{1}, q_{1}\right)$ $\leqq d\left(p_{r_{i}}, q_{r_{i}}\right)$. Therefore the supposition that the lemma was false was incorrect and there are two points $x$ and $y$ such that $d(x, y)>d\left(x_{1}, y_{1}\right)$.

Consider next the case in which both $p$ and $q$ are periodic, $p$ of period $m$ and $q$ of period $n$. For the product $m n, p_{m n}=p$ and $q_{m n}=q$. Therefore $d\left(p_{m n}, q_{m n}\right)=d(p, q)<d\left(p_{1}, q_{1}\right)$. If the distance between no two points is decreased, it follows that $d\left(p_{1}, q_{1}\right) \leqq d\left(p_{m n}, q_{m n}\right)$. Hence the distance between some two points must decrease.

The last case is that in which one of the points, say $p$, is periodic of period $m$ and $q$ is not periodic. The procedure in this case is much the same as in the first case. From the sequence $\left(q_{m}, q_{2 m}, q_{3 m}, \cdots\right)$ choose a subsequence $\left(q_{k_{1}}, q_{k_{2}}, q_{k_{s}}, \cdots\right)$ satisfying the Cauchy convergence criterion. As before it is possible to obtain a sequence $\left(q_{r_{1}}, q_{r_{2}}, q_{r_{8}}, \cdots\right)$ which converges 
to $q$. For all $n, r_{n}$ is a multiple of $m$, because $r_{n}=k_{j}-k_{i}$ and $k_{i}$ and $k_{j}$ are multiples of $m$. Therefore for all $n, p_{r_{n}}=p$, and $d\left(p_{r_{i}}, q_{r_{i}}\right)$ converges to $d(p, q)$ and a contradiction may be obtained as before.

CoROLlARY. If $E$ is a conditionally compact metric space and if $T$ is a one-to-one transformation of $E$ into itself which does not leave all distances invariant, then $T$ must increase at least one distance and decrease at least one distance.

If $T$ increases some distance, the conclusion follows immediately from the lemma. If $T$ decreases some distance, the lemma may be applied to the inverse of $T$ and the conclusion obtained in that manner.

A metric space is said to be arc-wise connected if every two points in the space may be joined by a simple arc which lies in the space. A simple arc is defined to be any set which is homeomorphic to the set of real numbers on the closed interval from zero to one. In the following theorem it is assumed that the space contains at least two points.

Theorem. If $E$ is an arc-wise connected conditionally compact metric space and if $T$ is a one-to-one continuous transformation of $E$ into itself, then there are two distinct points of $E$ whose distance is invariant under $T$.

If the distance between every pair of points is invariant, the theorem is obviously true. It may therefore be assumed that $T$ alters the distance between some pair of points. It follows from the corollary that there are two points $P$ and $Q$ such that $d(P, Q)<d\left(P_{1}, Q_{1}\right)$ and two points $R$ and $S$ such that $d(R, S)$ $>d\left(R_{1}, S_{1}\right)$. If $P$ and $R$ are coincident, $Q$ and $S$ can not be coincident, and if $Q$ and $S$ are coincident, $P$ and $R$ are not coincident. The notation may be so chosen that it is $P$ and $R$ which are not coincident. Then the points $Q$ and $S$ may or may not be coincident. There is an arc $P R$ joining $P$ and $R$ and lying in $E$. It is necessary to consider several cases and subcases.

Case I. The points $Q$ and $S$ are on $P R$ and are coincident. If $Q$ and $S$ are coincident, the two must be distinct from $P$ and from $R$. A homeomorphism may be established between $P Q$ and $Q R$ in which $P$ corresponds to $Q$ and $Q$ corresponds to 
$R$. If $x$ is any point of $P Q$, its corresponding point on $Q R$ is denoted by $x^{\prime}$. An essential fact is that $x$ and $x^{\prime}$ are never coincident. Define a real-valued function $f(x)$ for each point $x$ of $P Q$ as follows:

$$
f(x)=d\left(x, x^{\prime}\right)-d\left(x_{1}, x_{1}^{\prime}\right) .
$$

Since $f(P)=d(P, Q)-d\left(P_{1}, Q_{1}\right), f(P)$ is negative. Since $f(Q)=d(Q, R)-d\left(Q_{1}, R_{1}\right)=d(S, R)-d\left(S_{1}, R_{1}\right), f(Q)$ is positive. The function $f(x)$ is continuous and therefore for some point $M$ of $P R, f(M)=d\left(M, M^{\prime}\right)-d\left(M_{1}, M_{1}^{\prime}\right)=0$, and $M$ and $M^{\prime}$ are the two distinct points desired.

CASE II. The points $Q$ and $S$ are on $P R$ and are distinct.

Assume first that $Q$ coincides with $R$. Then $S$ can not coincide with $P$. Define a function $h(x)$ for all points $x$ of $\operatorname{arc} P S$ in the following manner:

$$
h(x)=d(x, R)-d\left(x_{1}, R_{1}\right) .
$$

The function $h(x)$ is continuous and, since $h(P)$ is negative and $h(S)$ is positive, there must be some point $M$ on $P S$ such that $h(M)=0$. The points $M$ and $R$ are then the required points.

If $S$ coincides with $P, Q$ can not coincide with $R$ and the procedure is the same as in the situation just completed.

If neither $Q$ nor $S$ coincides with either $R$ or $P$, there are again two possibilities. As the first possibility assume that $S$ separates $P$ and $Q$ on the $\operatorname{arc} P R$. In this case a homeomorphism may be set up between the arc $P S$ and the $\operatorname{arc} Q R$ in which $P$ corresponds to $Q, S$ corresponds to $R$, and in which no two corresponding points coincide. The proof may be completed as in Case I by a function $f(x)$ defined on $P S$. As the second possibility $Q$ may separate $P$ and $S$ on the arc $P R$. By proceeding with care it is also possible in this case to set up a homeomorphism between $P S$ and $Q R$ in which $P$ corresponds to $Q, S$ corresponds to $R$, and in which no corresponding points coincide. Again the proof may be completed by means of a function $f(x)$ defined on $P S$.

Case III. Either $Q$ or $S$ is not on $P R$.

As there is no difference between the two cases assume that 
$Q$ is not on $P R$. If $Q$ and $S$ are coincident, define a function $h(x)$ on $P R$ as follows:

$$
h(x)=d(x, Q)-d\left(x_{1}, Q_{1}\right) .
$$

This function is continuous; it is negative at $P$ and positive at $R$ and the proof is as before.

Suppose that $Q$ and $S$ are distinct and denote by $Q S$ the arc joining $Q$ and $S$. If $Q S$ includes both $P$ and $R$, the situation is essentially the same as that in which the arc $P R$ includes all four points. It is therefore assumed that $Q S$ does not contain both $P$ and $R$. Consider first the case in which $Q S$ does not include $R$. There is then a point $A$ on $P R$ which is not on $Q S$ and which is such that no point of $P R$ between $A$ and $R$ is on $Q S$. There is a point $B$ on $Q S$ such that $B$ is not on $P R$ and such that no point of $Q S$ between $Q$ and $B$ is on $P R$. If a homeomorphism between $P R$ and $Q S$ is set up in such a way that $P$ corresponds to $Q$, $A$ to $B$, and $R$ to $S$, then no two corresponding points are coincident. Therefore a function $f(x)$ may be used as in previous cases. The points $A$ and $B$ are so chosen that $A \neq R$ and $B \neq Q$.

The remaining possibility is that $Q S$ does not include $P$. If $S$ is not on $P R$, this reduces to the case just considered, where the parts played by the various points are interchanged. Assume, then, that $S$ is on $P R$. Let $K$ be the first point on $Q S$ which is a point of $P R$, the ordering on $Q S$ being assumed to begin at $Q$ and continue to $S$. If $K$ and $R$ are coincident, use may be made of a homeomorphism between $P S$ and $Q K$. No two points of such a homeomorphism are coincident. If $K$ is not $R$, form an arc $C$ by adding the subarc $Q K$ of $Q S$ to the subarc $K S$ of $P R$. In the event that $K$ and $S$ are coincident, $K S$ is not a subarc but merely a point. This does not alter that which is to follow. The arc $C$ joins $Q$ and $S$, and since $C$ does not contain $R$, the situation is reduced to one already treated.

HaRVARd UNIVERSITY 